\title{
The Effective Equation of Motion on the Brane World Gravity
}

\author{
F. P. Zen ${ }^{1}$, Arianto ${ }^{1,2}$, B. E. Gunara ${ }^{1} \&$ H. Zainuddin ${ }^{3}$ \\ ${ }^{1}$ Theoretical High Energy Physics Lab., Faculty of Mathematics and Natural Sciences, \\ Institute of Technology Bandung, Jl. Ganesha 10 Bandung 40132, Indonesia \\ ${ }^{2}$ Department of Physics, Udayana University, \\ Jl. Kampus Bukit Jimbaran Denpasar 80361, Indonesia \\ ${ }^{3}$ Theoretical Studies Laboratory, ITMA, Universiti Putra Malaysia, \\ 43400 UPM Serdang, Selangor, Malaysia
}

\begin{abstract}
Randall-Sundrum single brane system (RS II) is investigated systematically, with an emphasis of the effective equation of motion on the brane, by using a low energy expansion method (gradient expansion method) in the bulk. Through the junction condition, we deduce in the zero ${ }^{\text {th }}$ order fine tuning for RS model. The modified Einstein equation, as well as Friedmann equation with dark energy radiation on the brane is derived from the first order expansion. We also clarify the emergence of the AdS/CFT correspondence for the case of the higher order expansion in a low energy iteration scheme.
\end{abstract}

Keywords: Brane World Gravity; Einstein Equation; Dark Energy; AdS/CFT.

\section{Introduction}

One of the long standing problem in particle physics and gravitational theories is how to understand quantum theory of gravity. Nowadays, the only possible candidate for this theory is the superstring theory ${ }^{[1]}$. Interestingly, this theory predicts the existence of the extra dimensions. In order to reconcile this prediction with our observed four dimensional universe, we need a mechanism to compactify the extra dimensions. In particular the setup of heterotic M-theory and its compactification down to five dimensions ${ }^{[2]}$ leads to a well motivated five dimensional brane world scenario, which can be used to study its consequences in particle physics and cosmology ${ }^{[3]}$.

Randall and Sundrum (RS) proposed two similar but distinct phenomenological brane world scenarios ${ }^{[4,5]}$. The first scenario is composed of two branes of the opposite tension, so called RS I. This scenario is the five dimensional spacetime which all matter fields are assumed to be confined on branes at fixed points of the $S^{1} / Z_{2}$ so that the bulk is described by pure Einstein gravity with a negative cosmological constant. On the other hand, the second scenario has a single brane with a positive tension, so called RS II. The fifth dimension is

Makalah diterima redaksi tanggal 4 Oktober 2005, revisi diterima tanggal 9 Januari 2006. 
infinite but still $\mathrm{Z}_{2}$ symmetry is imposed. In both scenarios the existence of the branes and the bulk cosmological constant makes the bulk geometry curved, or warped.

Furthermore, the brane world models are expected to shed some light onto not only quantum gravity and unification issues, but also cosmological issues such as the cosmological constant or dark energy problem, or even particle physics ones such as the hierarchy problem. There is also a brane world alternative to the standard big bang plus inflation scenario ${ }^{[6,7]}$. This scenario consist of a five dimensional bulk bounded by two branes, which are as usual located at the fixed point of the $\mathrm{S}^{1} / \mathrm{Z}_{2}$ orbifold. Although many brane world models might be over simplified, they should help in learning and understanding the properties of an effective theory derived from some action in space-time dimensions $\mathrm{d}>4{ }^{[8]}$.

There are two approaches to obtain the effective Einstein equations on the brane in the context of Randall-Sundrum scenarios, namely covariant curvature formulation and gradient expansion method. In the covariant formulation ${ }^{[9]}$, the effective Einstein equation can be obtained by projecting the covariant five dimensional Einstein equations onto brane. The resulting projected equations are modified with respect to general relativity due to the presence of a local quadratic term in the sources and to the presence of a non-local term which is the projection of the five dimensional Weyl tensors. This last term carries information of the bulk gravitational field on the brane and its contribution is of fundamental importance as it might be relevant even at low energy ${ }^{[10,11]}$.

The main difficulty in understanding the contribution of the projected Weyl tensor to the effective theory on the brane is in its non-local character. The equation for the projected Weyl tensor on the brane are not closed so that solving the full five dimensional equation of motion is necessary.

On the other hand, gradient expansion method gives a way out of this problem $^{[12]}$. The main idea is to treat the issue perturbatively, defining a low energy regime in which the energy density on the brane is kept small with respect to bulk vacuum energy density. The perturbation parameter is defined as the ratio between these two energy densities. The five dimensional equations of motion can be solved at different orders in the perturbation parameter. This method allows in principle to derive the effective equations of motion on the brane at each order.

In this paper, we investigate more systematic the effective equations of motion on the brane in the context of RS II by using gradient expansion method in the 
bulk. By performing a perturbative expansion of the metric, an expansion of the extrinsic curvature tensor and Weyl tensor are considered. The four basic equations for the five dimensional evolution equations and junction conditions are then solved at different order in the expansion parameter. The parameter of expansion is determined as in the Anti de Sitter (AdS) scenario. There is a constant scale, namely the AdS curvature scale, to which quantities can be compared.

The paper is organized as follows. In section 2, we review a covariant curvature formulation to obtain the effective Einstein equation on the brane. In section 3, we give the basic formulation of the gradient expansion method by solving five dimensional equations of motion and imposing the Dirichlet boundary condition at the brane position. In section 4 we derive the effective equations of motion on the brane. We conclude our result in section 5 .

\section{The Covariant Curvature Tensor Approach}

In this section we show how to recover the effective Einstein equations on a four dimensional hypersurface, the brane, which is embedded in a five dimensional space-time, the bulk ${ }^{[9]}$.

\subsection{The Einstein Equation on the Brane}

The covariant derivative $D_{A}(\mathrm{~A}=0,1,2,3,5)$ associated with the induced metric $h_{A B}$ has the following expression

$$
D_{C} T_{B_{1} \cdots B_{l}}^{A_{1} \cdots A_{k}}=h_{D_{1}}^{A_{1}} \cdots h_{B_{l}}^{E_{l}} h_{C}^{F} \nabla_{F} T_{E_{1} \cdots E_{l}}^{D_{1} \cdots D_{k}} .
$$

So that, for example,

$$
D_{B} \omega_{C}=h_{B}^{D} h_{C}^{E} \nabla_{D} \omega_{E},
$$

where $\nabla_{A}$ is the covariant derivative with respect to the five dimensional metric. The action of the four dimensional Riemann tensor on a 1-form is given by

$$
{ }^{(4)} R_{A B C}{ }^{D} \omega_{D}=\left[D_{A}, D_{B}\right] \omega_{C}=D_{A} D_{B} \omega_{C}-D_{B} D_{A} \omega_{C},
$$

and the right hand side can be computed from

$$
\begin{aligned}
& D_{A} D_{B} \omega_{C}=D_{A} h_{B}{ }^{E} h_{C}^{D} \nabla_{D} \omega_{E} \\
& =h_{A}{ }^{F} h_{B}{ }^{G} h_{C}{ }^{K} \nabla_{F} T_{G K}=h_{A}{ }^{F} h_{B}{ }^{G} h_{C}{ }^{K} \nabla_{F}\left(h_{G}{ }^{D} h_{K}{ }^{E} \nabla_{D} \omega_{E}\right)
\end{aligned}
$$




$$
\begin{aligned}
& =h_{A}^{F} h_{B}{ }^{G} h_{C}{ }^{K}\left[\left(\nabla_{F} h_{G}^{D}\right) h_{K}{ }^{E} \nabla_{D} \omega_{E}+h_{G}^{D}\left(\nabla_{F} h_{K}{ }^{E}\right) \nabla_{D} \omega_{E}+h_{G}{ }^{D} h_{K}{ }^{E}\left(\nabla_{F} \nabla_{D} \omega_{E}\right)\right] \\
& =-K_{A B} h_{C}{ }^{E} n^{D} \nabla_{D} \omega_{E}-K_{A C} n^{E} h_{B}^{D} \nabla_{D} \omega_{E}+h_{A}^{F} h_{B}{ }^{D} h_{C}{ }^{E}\left(\nabla_{F} \nabla_{D} \omega_{E}\right)
\end{aligned}
$$

where $K_{A B}$ is the extrinsic curvature tensor defined by

$$
K_{A B}=h_{A}{ }^{C} h_{B}^{D} \nabla_{C} n_{D} .
$$

When antisymmetrizing over $A$ and $B$ and considering that the extrinsic curvature tensor is symmetric we have

$$
{ }^{(4)} R_{A B C}{ }^{D} \omega_{D}=-\left(-K_{A C} K_{B}^{E}+K_{B C} K_{A}{ }^{E}\right) \omega_{E}+h_{A}{ }^{F} h_{B}{ }^{G} h_{C}{ }^{K}\left[\nabla_{F}, \nabla_{G}\right] \omega_{K} .
$$

Finally, using the definition of the five dimensional Riemann tensor

$$
{ }^{(5)} R_{A B C D} \omega^{D}=\nabla_{A} \nabla_{B} \omega_{C}-\nabla_{B} \nabla_{A} \omega_{C},
$$

we obtain

$$
{ }^{(4)} R_{A B C}{ }^{D}=h_{A}^{F} h_{B}^{G} h_{C}{ }^{K} h_{J}^{D}{ }^{(5)} R_{F G K}{ }^{J}+K_{A C} K_{B}^{D}-K_{B C} K_{A}{ }^{D} .
$$

The equation (2.8) is called Gauss equation. This equation provide a link between the five dimensional Riemann tensor with the four dimensional one on the brane. Contracting the former equation over the indices $B$ and $D$, we obtain the expression for the Ricci tensor and the Ricci scalar

$$
\begin{aligned}
& { }^{(4)} R_{A C}=h_{A}^{F} h_{B}{ }^{G} h_{C}{ }^{K} h_{J}^{B}{ }^{(5)} R_{F G K}+K_{A C} K-K_{C B} K_{A}{ }^{B}, \\
& { }^{(4)} R={ }^{(5)} R-2 n^{G} n^{J}{ }^{(5)} R_{G J}+K^{2}-K_{A B} K^{A B} .
\end{aligned}
$$

By inserting the equations (2.9) and (2.10) into the four dimensional Einstein tensor

$$
{ }^{(4)} G_{A C}={ }^{(4)} R_{A C}-\frac{1}{2} h_{A C}{ }^{(4)} R,
$$

we can write down the Einstein tensor on the brane

$$
\begin{gathered}
{ }^{(4)} G_{A C}={ }^{(5)} G_{M R} h_{A}^{M} h_{C}^{R}-{ }^{(5)} R_{M N R S} h_{A}^{M} n^{N} h_{C}^{R} n^{S}+h_{A C} n^{G} n^{J}{ }^{(5+n)} R_{G J} \\
+K_{A C} K-K_{A}^{B} K_{B C}-\frac{1}{2} h_{A C}\left(K^{2}-K_{B D} K^{B D}\right) .
\end{gathered}
$$

Furthermore, we can always decompose the Riemann tensor in five dimensions into the Weyl tensor, the Ricci tensor and the Ricci scalar which gives ${ }^{[13]}$ 


$$
\begin{aligned}
{ }^{(5)} R_{M N R S}={ }^{(5)} C_{M N R S}+\frac{1}{3}\left[{ }^{(5)} R_{M R} g_{N S}-{ }^{(5)} R_{M S} g_{N R}+{ }^{(5)} R_{N S} g_{M R}-{ }^{(5)} R_{N R} g_{M S}\right] \\
-\frac{1}{12}{ }^{(5)} R\left[g_{M R} g_{N S}-g_{M S} g_{N R}\right] .
\end{aligned}
$$

Using this decomposition, the equation (2.12) and five dimensional Einstein equations

$$
{ }^{(5)} G_{A B}={ }^{(5)} R_{A B}-\frac{1}{2} g_{A B}{ }^{(5)} R=\kappa_{5}^{2} T_{A B},
$$

we obtain

$$
\begin{aligned}
{ }^{(4)} G_{A B}=\frac{2 \kappa_{5}^{2}}{3} & {\left[h_{A}^{C} h_{B}^{D} T_{C D}+\left(n^{C} n^{D} T_{C D}-\frac{1}{4} T\right) h_{A B}\right] } \\
& +K K_{A B}-K_{A}^{C} K_{B C}-\frac{1}{2} h_{A B}\left(K^{2}-K^{C D} K_{C D}\right)-E_{A B},
\end{aligned}
$$

where $T_{A B}$ is the five-dimensional energy-momentum tensor which in general takes into account brane and bulk contribution. We also have defined the projection of the five- dimensional Weyl tensor as

$$
E_{A B}=C_{M N R S} n^{M} n^{R} h_{A}^{N} h_{B}^{S} .
$$

Additionally, we have the Codazzi equation, which can be obtained in a same way as for Gauss equation (2.8),

$$
D_{B} K_{A}^{B}-D_{A} K=\kappa_{D}^{2} T_{E F} h_{A}^{E} n^{F} .
$$

In the following we make further assumptions which allow us to interpret equation (2.15) on the brane. As an original RS scenario, we choose $Z_{2}$ symmetry across the brane.

\subsection{The Junction Conditions}

In order to know about the local geometry due to some matter distribution, we start with the five dimensional Einstein equations (2.14) which can be written into

$$
{ }^{(5)} R_{A B}=\kappa_{5}^{2}\left(T_{A B}-\frac{1}{3} g_{A B} T\right),
$$


where $T$ denotes the trace of $T_{A B}$. Assume that the brane has a finite thickness $\varepsilon$ and let $\Sigma^{-}$and $\Sigma^{+}$denote the two boundaries of the brane $\sum$. Furthermore, we adopt Gaussian normal coordinates $y$, in which the brane is located at a fixed point $y=0$ on $\Sigma^{-}$and $y=\varepsilon$ on $\Sigma^{+}$. In the Gaussian normal coordinates, the five dimensional Ricci tensor projected onto the brane can be written as ${ }^{[9]}$

$$
{ }^{(5)} R_{M R} h_{A}^{M} h_{C}^{R}={ }^{(4)} R_{A C}-\text { Lie }_{n} K_{A C}+2 K_{A N} K_{C}^{N}-K_{A C} K,
$$

where $\mathrm{Lie}_{\mathrm{n}}$ is Lie derivative in direction of the unit normal $n$. Inserting equation (2.18) into equation (2.19) and both sides are integrated across $\sum$ we have

$$
\int_{y=0}^{y=\varepsilon} d y \kappa_{5}^{2}\left(T_{M N} h_{A}^{M} h_{B}^{N}-\frac{1}{3} T h_{A B}\right)=\int_{y=0}^{y=\varepsilon} d y \frac{\partial K_{A B}}{\partial y}+\int_{y=0}^{y=\varepsilon} d y\left(-K K_{A B}+2 K_{A C} K_{B}^{C}+{ }^{(4)} R_{A B}\right)
$$

We see that on the right hand side, all tensor are by definition tangential, whereas on the left hand side, the bulk energy momentum tensor and the bulk metric have to be projected onto each brane in the integration domain. It is reasonable to expect that the second term on the right hand side is bounded between $\Sigma^{-}$and $\Sigma^{+}$, therefore the integral vanishes in the limit $\varepsilon \rightarrow 0$. We obtain

$$
\lim _{\varepsilon \rightarrow 0} \int_{y=0}^{y=\varepsilon} d y \kappa_{5}^{2}\left(T_{M N} h_{A}^{M} h_{B}^{N}-\frac{1}{3} T h_{A B}\right)=K_{A B}(y=\varepsilon)-K_{A B}(y=0) \equiv K_{A B}^{+}-K_{A B}^{-} .
$$

We define an energy-momentum tensor on the brane $\Sigma$

$$
\bar{T}_{A B} \equiv \lim _{\varepsilon \rightarrow 0} \int_{0}^{\varepsilon} d y T_{M N} h_{A}^{M} h_{B}^{N},
$$

such that the equation (2.21) becomes

$$
K_{A B}^{+}-K_{A B}^{-}=\kappa_{5}^{2}\left(\bar{T}_{A B}-\frac{1}{3} h_{A B} \bar{T}\right) .
$$

The $Z_{2}$ symmetry gives the following additional condition

$$
K_{A B}^{+}=-K_{A B}^{-} \equiv K_{A B},
$$

which is allow us to compute (2.15) by evaluating the tensors on one side or the other of the brane. Using equation (2.23), we obtain 


$$
\begin{aligned}
& K_{\mu \nu} K=-\frac{\kappa_{5+n}^{4}}{4}\left(\frac{1}{3} \bar{T}_{\mu \nu} \bar{T}-\frac{1}{9} \bar{T}^{2} h_{\mu \nu}\right), \\
& K_{\mu}^{\rho} K_{v \rho}=\frac{\kappa_{5}^{4}}{4}\left(\bar{T}_{\mu}^{\rho} \bar{T}_{v \rho}-\frac{2}{3} \overline{T T}_{\mu \nu}+\frac{1}{9} \bar{T}^{2} h_{\mu \nu}\right), \\
& -\frac{1}{2} h_{\mu \nu}\left(K^{2}-K^{\rho \sigma} K_{\rho \sigma}\right)=-\frac{\kappa_{5}^{4}}{8} h_{\mu \nu}\left(\frac{1}{3} \bar{T}^{2}-\bar{T}_{\rho \sigma} \bar{T}^{\rho \sigma}\right) .
\end{aligned}
$$

We denote the four dimensional quantities on the brane by indices $\mu, v(\mu, v=0$, 1, 2, 3), for example $\bar{T}_{\mu \nu} \equiv\left(\bar{T}_{A B}\right)_{\mu \nu}$. Then we obtain the effective Einstein equation on the brane supplemented by the junction conditions in which the extrinsic curvature is determined by brane matter

$$
{ }^{(4)} G_{\mu \nu}=-\frac{\kappa_{5}^{2}}{2} \Lambda_{5} h_{\mu \nu}-\frac{\kappa_{5}^{4}}{4}\left(-\frac{1}{3} \overline{T T}_{\mu \nu}+\bar{T}_{\mu}^{\rho} \bar{T}_{\nu \rho}\right)-\frac{\kappa_{5}^{4}}{4}\left(\frac{1}{6} \bar{T}^{2}-\frac{1}{2} \bar{T}_{\rho \sigma} \bar{T}^{\rho \sigma}\right) h_{\mu \nu}-E_{\mu \nu} .
$$

This equation is not standard Einstein equation because it contains terms quadratic in the matter source and the Weyl tensor, $E_{\mu \nu}$. In the above Einstein equations, the matter source is represented by the term $\prod_{\mu v}$ (see eq. (2.33)), which is quadratic in the energy-momentum tensor. The origin of the quadratic behavior traces back to the Gauss equation (2.8). The Weyl tensor, $E_{\mu \nu}$, is a projection of the five-dimensional Weyl tensor onto the brane. Unfortunately, it cannot be determined from internal brane quantities alone. The Weyl tensor, $E_{\mu \nu}$, contains information about the bulk but is constrained by the matter on the brane. Therefore $E_{\mu \nu}$ is a non-local quantity. Furthermore, the Weyl tensor represents 5-dimensional gravitational waves propagating in the bulk, and so $E_{\mu v}$ corresponds to their energy-momentum deposited on the brane. When the Friedmann equation is calculated, this contribution shows up as $C / a^{4}$ (see eq. (4.4)).

We now decompose the energy-momentum tensor $\bar{T}_{\mu \nu}$ on the brane into a tension part $\lambda$ and a matter fields part, so that

$$
\bar{T}_{\mu \nu}=-\lambda h_{\mu \nu}+T_{\mu v} .
$$

Then we obtain 


$$
{ }^{(4)} G_{\mu v}=-\Lambda_{4} h_{\mu \nu}+8 \pi G_{4} T_{\mu v}+\kappa_{5}^{4} \prod_{\mu \nu}-E_{\mu v}
$$

where

$$
\begin{aligned}
& \Lambda_{4}=\frac{1}{2} \kappa_{5}^{2}\left[\Lambda_{5}+\frac{1}{6} \kappa_{5}^{2} \lambda^{2}\right] \\
& G_{4}=\frac{\kappa_{5}^{4} \lambda}{48 \pi} \\
& \prod_{\mu \nu}=\frac{1}{4}\left(\frac{1}{3} T T_{\mu \nu}-T_{\mu}^{\rho} T_{v \rho}-\frac{1}{6} T^{2} h_{\mu \nu}+\frac{1}{2} T_{\rho \sigma} T^{\rho \sigma} h_{\mu \nu}\right) .
\end{aligned}
$$

We see that the effective cosmological constant $\Lambda_{4}$ on the brane is given by a combination of the brane tension and the bulk cosmological constant $\Lambda_{5}$. In order to obtain viable solution, we need fine-tuning, by which brane worlds open up opportunities for a new approaches to the cosmological constant problem. Furthermore, we see that Newton's constant $G_{4}$ on the brane depends linearly on the tension brane. Therefore, it is important that we live on the positive tension brane. The term $\prod_{\mu \nu}$, which is quadratic term in the $\tau_{\mu \nu}$ on the brane can change the cosmological evolution significantly.

The important result obtained in equation (2.30) is that the four dimensional Einstein equations are modified with respect to general relativity in those two additional terms appear: a local quadratic term in the source, which becomes relevant at high energy and a non-local term $E_{\mu v}$, which corresponds to the projection on the brane of the five- dimensional Weyl tensor. If no scalar fields or other fields are considered in the bulk, equations (2.17) and (2.23) imply the conservation law for the brane energy-momentum tensor $D^{\mu} \tau_{\mu \nu}=0$. Then the derivative of the projection Weyl tensor $E_{\mu \nu}$ is given by

$$
D^{\mu} E_{\mu \nu}=\frac{1}{4} \kappa_{5}^{4}\left(\frac{1}{3}\left(T_{\mu \nu}-T h_{\mu \nu}\right) D^{\mu} T+T^{\rho \sigma}\left(D_{v} T_{\rho \sigma}-D_{\sigma} T_{\nu \rho}\right)\right) .
$$

\section{The Gradient Expansion Method}

In this section we derive the low energy expansion method (gradient expansion method) first proposed by Kanno and Soda ${ }^{[12]}$ to study the Randall-Sundrum II scenario. In this method, the full five dimensional equations of motion are solved, at different orders, in the bulk by performing a perturbation expansion in 
the metric. The parameter of expansion is defined so that the low energy regime corresponds to a regime in which the energy density on the brane is smaller then the brane tension $(\rho<<\lambda)$.

\subsection{The Parameter of Expansion}

The parameter of expansion can be expressed as follows

$$
\varepsilon=\left(\frac{l}{L}\right)^{2}
$$

where $l=l_{A d S}$ is the bulk curvature scale and $L$ is the brane curvature scale. Then the extrinsic curvature is expanded as

$$
K_{\mu}^{v}=K_{\mu}^{(0) v}+\varepsilon K_{\mu}^{(1) v}+\varepsilon^{2} K_{\mu}^{(2) v}+\cdots .
$$

The iteration scheme consists in writing the metric $g_{\mu v}$ as a sum of local tensors built out of the induced metric on the brane $h_{\mu \nu}$,

$$
d s^{2}=a^{2}(y)\left[h_{\mu \nu}\left(x^{\mu}\right)+g_{\mu \nu}^{(1)}\left(y, x^{\mu}\right)+g_{\mu \nu}^{(2)}\left(y, x^{\mu}\right)+g_{\mu \nu}^{(2)}\left(y, x^{\mu}\right)+\cdots\right] d x^{\mu} d x^{v}+d y^{2},
$$

where $a(y)$ is a warp factor as a function of fifth dimension, and we also impose the Dirichlet boundary condition at the brane position

$$
g_{\mu \nu}^{(i)}\left(y=0, x^{\mu}\right)=0, \quad i=1,2, \ldots
$$

In the Gaussian normal coordinate system, the geometry of the brane world is described by

$$
d s^{2}=g_{\mu \nu}\left(y, x^{\mu}\right) d x^{\mu} d x^{v}+d y^{2} .
$$

In order to obtain the effective equations of motion, we need first to find the equation of motion of five dimensional Einstein equations and the junction condition. The four basic equations which hold in the bulk are ${ }^{[12]}$

$$
\begin{aligned}
& \partial_{y} \sum_{\mu}^{v}-K \sum_{\mu}^{v}=-\left[{ }^{(4)} R_{\mu}^{v}-\frac{1}{4} \delta_{\mu}^{\nu}{ }^{(4)} R\right], \\
& \partial_{y} K-\sum^{\mu \nu} \sum_{\mu \nu}-\frac{1}{4} K^{2}=-\frac{4}{l^{2}},
\end{aligned}
$$




$$
\begin{aligned}
& \frac{3}{4} K^{2}-\sum^{\mu v} \sum_{\mu v}=\left[{ }^{(4)} R\right]+\frac{12}{l^{2}}, \\
& D_{v} \sum_{\mu}^{v}-\frac{3}{4} D_{\mu} K=0,
\end{aligned}
$$

where we decompose the extrinsic curvature into the traceless part and the trace part

$$
\begin{aligned}
& K_{\mu}^{v}=\sum_{\mu}^{v}+\frac{1}{4} \delta_{\mu}^{v} K, \\
& K=-\frac{\partial}{\partial y} \log \sqrt{-g} .
\end{aligned}
$$

The junction condition is given by

$$
\left.\left[K_{\mu}^{v}-\delta_{\mu}^{v} K\right]\right|_{y=0}=\frac{\kappa_{5}^{2}}{2}\left(-\lambda \delta_{\mu}^{v}+T_{\mu \nu}\right) .
$$

\subsection{Zeroth Order}

At zeroth order matter is neglected, we intend vacuum brane, and going at higher orders means we are considering perturbation of the vacuum solution as matter is added to the brane. The equations to solve are

$$
\begin{aligned}
& \partial_{y} \sum_{\mu}^{(0) v}-K^{(0)} \sum_{\mu}^{(0) v}=0 \\
& \partial_{y} K^{(0)}-\sum^{(0) \mu v} \sum_{\mu \nu}^{(0)}-\frac{1}{4} K^{(0) 2}=-\frac{4}{l^{2}}, \\
& \frac{3}{4} K^{(0) 2}-\sum^{(0) \mu v} \sum_{\mu \nu}^{(0)}=\frac{12}{l^{2}} \\
& D_{v} \sum_{\mu}^{(0) v}-\frac{3}{4} D_{\mu} K^{(0)}=0 .
\end{aligned}
$$

Integrating equation (3.9a) and using the constrain (3.9d) we see that

$$
\sum_{\mu}^{v(0)}\left(y, x^{\mu}\right)=\frac{C_{\mu}^{v}\left(x^{\mu}\right)}{\sqrt{-g}},
$$


is a solution for equation (3.9a). The tensor field $C_{\mu}^{v}\left(x^{\mu}\right) \equiv \sum_{\mu}^{v}\left(y=0, x^{\mu}\right)$ is the integration constant. The Codazzi equation gives a constraint (3.9d) and requiring $D_{v} C_{\mu}^{v}\left(x^{\mu}\right)=0$. We put $C_{\mu}^{v}\left(x^{\mu}\right)=0$ and the equation (3.9c) gives

$$
K^{(0)}=\frac{4}{l} \text {. }
$$

From the definition of the extrinsic curvature,

$$
K_{\mu \nu}^{(0)}=-\frac{1}{2} \partial_{y} g_{\mu \nu}^{(0)}
$$

and using the equation (3.7) we obtain the metric at zero order,

$$
\begin{aligned}
& g_{\mu \nu}^{(0)}\left(y, x^{\mu}\right)=\exp \left(-\frac{2}{l} y\right) h_{\mu \nu}\left(x^{\mu}\right), \\
& d s^{2}=a^{2}(y) h_{\mu \nu}\left(x^{\mu}\right) d x^{\mu} d x^{\nu}+d y^{2}, a(y)=\exp \left(-\frac{1}{l} y\right) .
\end{aligned}
$$

The tensor $h_{\mu v}$ is a tensor field depending only on the brane coordinates, so called the induced metric.

On the 3-brane we have the following junction condition

$$
\left[K_{\mu}^{(0) v}-\delta_{\mu}^{v} K^{(0)}\right]_{y=0}=-\frac{\kappa_{5}^{2}}{2} \lambda \delta_{\mu}^{v} .
$$

Then we get the relation

$$
\frac{\kappa_{5}^{2}}{6} \lambda=\frac{1}{l}
$$

We see that the equation (3.15) is the fine tuning condition of the RandallSundrum II model.

\subsection{First Order}

At the next order solution is obtained by taking into account the terms neglected at zero order. The aim of this subsection is now to solve the four basic equations (3.6a) - (3.6d). At first order we have 


$$
\begin{aligned}
& \partial_{y} \sum_{\mu}^{(1) v}-\frac{4}{l} \sum_{\mu}^{(1) v}=-\left[{ }^{(4)} R_{\mu}^{v}-\frac{1}{4} \delta_{\mu}^{\nu}{ }^{(4)} R\right]^{(1)}, \\
& \frac{6}{l} K^{(1)}=\left[{ }^{(4)} R\right]^{(1)} \\
& \partial_{y} K^{(1)}-\frac{2}{l} K^{(1)}=0 \\
& \tilde{D}_{\nu} \sum_{\mu}^{(1) v}-\frac{3}{4} \tilde{D}_{\mu} K^{(1)}=0
\end{aligned}
$$

where the superscript (1) represents the order of the derivative expansion and $\tilde{D}_{\mu}$ denotes the covariant derivative with respect to $h_{\mu v}$. Taking into account of the metric $g_{\mu \nu}^{(0)}\left(y, x^{\mu}\right)=a^{2}(y) h_{\mu \nu}\left(x^{\mu}\right)$ yields

$$
\left[{ }^{(4)} R_{\mu}^{v}\right]^{(1)}=\frac{1}{a^{2}}{ }^{(4)} R_{\mu}^{v}(h), \quad\left[{ }^{(4)} R\right]^{(1)}={\frac{1}{a^{2}}}^{(4)} R(h) .
$$

It is straightforward to see that

$$
K^{(1)}=\frac{l}{6 a^{2}}{ }^{(4)} R(h),
$$

is a solution for equation (3.16b). Integration of equation (3.16a) gives the traceless part of the extrinsic curvature in this order, namely

$$
\sum_{\mu}^{v(1)}\left(y, x^{\mu}\right)=\frac{l}{2 a^{2}}\left({ }^{(4)} R_{\mu}^{v}(h)-\frac{1}{4} \delta_{\mu}^{v}(4) R(h)\right)+\frac{\chi_{\mu}^{v}\left(x^{\mu}\right)}{a^{4}},
$$

where the homogenous solution satisfies $\chi_{\mu}^{\mu}$ and $\tilde{D}_{v} \chi_{\mu}^{v}=0$. The second term of the equation (3.19) is essential to understanding the origin of the dark radiation from the holographic point of view. The metric at the first order reads

$$
g_{\mu \nu}^{(1)}=-\frac{l^{2}}{2}\left(\frac{1}{a^{2}}-1\right)\left({ }^{(4)} R_{\mu \nu}(h)-\frac{l}{6} h_{\mu \nu}{ }^{(4)} R(h)\right)-\frac{l}{2}\left(\frac{1}{a^{4}}-1\right) \chi_{\mu \nu},
$$

where we have used the trace part of the extrinsic curvature

$$
K_{\mu}^{(1) v}=\frac{l}{2) a^{2}}\left({ }^{(4)} R_{\mu}^{v}(h)-\frac{1}{6} \delta_{\mu}^{v(4)} R(h)\right)+\frac{\chi_{\mu}^{v}}{a^{4}} .
$$


The Effective Equation of Motion on the Brane World Gravity 13

\subsection{Second Order}

At second order, we have four basic equations,

$$
\begin{aligned}
& \partial_{y} \sum_{\mu}^{(2) \nu}-\frac{4}{l} \sum_{\mu}^{(2) v}=-\left[{ }^{(4)} R_{\mu}^{v}-\frac{1}{4} \delta_{\mu}^{\nu}{ }^{(4)} R\right]^{(2)}+K^{(1)} \sum_{\mu}^{(1) \nu}, \\
& K^{(2)}=\frac{l}{6}\left\{-\frac{3}{4} K^{(1) 2}+\sum^{\mu v(1)} \sum_{\mu \nu}^{(1)}+\left[{ }^{(4)} R\right]^{(2)}\right\}, \\
& \partial_{y} K^{(2)}-\frac{2}{l} K^{(2)}=\frac{1}{4} K^{(1) 2}+\sum^{\mu v(1)} \sum_{\mu \nu}^{(1)}, \\
& D_{\nu} \sum_{\mu}^{v}+\Gamma_{v \alpha}^{\alpha} \sum_{\mu}^{v}-\Gamma_{\mu \alpha}^{v} \sum_{\nu}^{\alpha}-\frac{3}{4} D_{\mu} K=0 .
\end{aligned}
$$

The junction condition at this order is

$$
\left.\left[K_{\mu}^{(2) v}-\delta_{\mu}^{v} K^{(2)}\right]\right|_{y=0}=\frac{\kappa_{5}^{2}}{2} T_{\mu}^{v} .
$$

Substituting solution up to the first order into the Ricci tensor and picking up the second order, we obtain

$$
\begin{aligned}
& {\left[{ }^{(4)} R_{\mu}^{v}-\frac{1}{4} \delta_{\mu}^{v}{ }^{(4)} R\right]^{(2)}=\frac{l^{2}}{2}\left(\frac{1}{a^{4}}-\frac{1}{a^{2}}\right)\left[-\frac{1}{2}\left(D^{\sigma} D_{\mu}{ }^{(4)} R_{\sigma}^{v}+D^{\sigma} D^{v(4)} R_{\mu \sigma}\right)\right.} \\
& +\frac{1}{3} D^{v} D_{\mu}{ }^{(4)} R+\frac{1}{2} D^{\sigma} D_{\sigma}{ }^{(4)} R_{\mu}^{v}-\frac{1}{12} \delta_{\mu}^{v} D^{\sigma} D_{\sigma}{ }^{(4)} R \\
& \left.+{ }^{(4)} R^{v \alpha\left({ }^{(4)}\right.} R_{\mu \alpha}-\frac{1}{6}{ }^{(4)} R_{\mu}^{v}{ }^{(4)} R-\frac{1}{4} \delta_{\mu}^{v}\left({ }^{(4)} R^{\alpha \beta}{ }^{(4)} R_{\alpha \beta}-\frac{1}{6}{ }^{(4)} R^{2}\right)\right],(3.24) \\
& {\left[{ }^{(4)} R\right]^{(2)}=\frac{l^{2}}{2}\left(\frac{1}{a^{4}}-\frac{1}{a^{2}}\right)\left({ }^{(4)} R^{\mu \nu}{ }^{(4)} R_{\mu \nu}-\frac{1}{6}{ }^{(4)} R^{2}\right) .}
\end{aligned}
$$

In this order, the solution of the tracelees part and the trace part of the extrinsic curvature are given by

$\sum_{\mu}^{v(2)}\left(y, x^{\mu}\right)=-\frac{l^{2}}{2}\left(\frac{y}{a^{4}}+\frac{l}{2 a^{2}}\right) S_{\mu}^{\nu}-\frac{l^{3}}{24 a^{2}}\left({ }^{(4)} R_{\mu}^{v}{ }^{(4)} R-\frac{1}{4} \delta_{\mu}^{\nu}{ }^{(4)} R^{2}\right)+\frac{l^{3}}{a^{4}} t_{\mu}^{\nu}\left(x^{\mu}\right)$, 
$K^{(2)}=\frac{l^{3}}{4 a^{4}}\left({ }^{(4)} R_{\mu \nu}{ }^{(4)} R^{\mu \nu}-\frac{2}{9}{ }^{(4)} R^{2}\right)-\frac{l^{3}}{12 a^{2}}\left({ }^{(4)} R_{\mu \nu}{ }^{(4)} R^{\mu \nu}-\frac{1}{6}{ }^{(4)} R^{2}\right)$,

where,

$$
\begin{aligned}
S_{\mu}^{\nu}= & { }^{(4)} R_{\nu}^{\beta}{ }^{(4)} R_{\mu \beta}-\frac{1}{3}{ }^{(4)} R^{(4)} R_{\mu}^{v}-\frac{1}{4} \delta_{\mu}^{\nu}{ }^{(4)} R^{\alpha \beta}{ }^{(4)} R_{\alpha \beta}+\frac{1}{12} \delta_{\mu}^{\nu}\left({ }^{(4)} R\right)^{2} \\
& -\frac{1}{2}\left[D_{\mu} D_{\sigma}{ }^{(4)} R^{\sigma V}+D^{\nu} D_{\sigma}{ }^{(4)} R_{\mu}^{\sigma}-\frac{2}{3} D_{\mu} D^{\nu}\left({ }^{(4)} R\right)\right] \\
& -\frac{1}{2}\left[-D^{\sigma} D_{\sigma}{ }^{(4)} R_{\mu}^{v}-\frac{1}{6} \delta_{\mu}^{V} D^{\alpha} D_{\alpha}^{(4)} R\right],
\end{aligned}
$$

is a transverse tensor and a traceless tensor $t_{\mu}^{\nu}$ is defined by

$$
t_{\mu}^{v}=\frac{1}{l^{3}} \sum_{\mu}^{v(2)}(y=0)
$$

\section{The Effective Equation of Motion on the Brane}

We are now able to write the effective Einstein equation in terms of the energy momentum tensors on the brane. To do so, we use the junction condition on a 3brane. At the first order we have

$$
\left.\left[K_{\mu}^{(1) v}-\delta_{\mu}^{v} K^{(1)}\right]\right|_{y=0}=\frac{\kappa_{5}^{2}}{2} T_{\mu}^{v}
$$

With this condition, the effective equation on the brane becomes

$$
\left({ }^{(4)} R_{\mu}^{v}(h)-\frac{1}{2} \delta_{\mu}^{\nu(4)} R(h)\right)=\frac{\kappa_{5}^{2}}{l} T_{\mu}^{v}-\frac{2}{l} \chi_{\mu}^{v}\left(x^{\mu}\right),
$$

and we can obtain the projective Weyl tensor as $E_{\mu}^{v}=2 \chi_{\mu}^{v} / l$. Then the effective Einstein equation (4.2)

$$
{ }^{(4)} G_{\mu}^{v}(h)=\frac{\kappa_{5}^{2}}{l} T_{\mu}^{v}-E_{\mu}^{v} .
$$

We see that the four dimensional Einstein equations are modified with respect to general relativity in that an additional term appear namely a non-local term 
$E_{\mu v}$, which corresponds to the projection on the brane of the five dimensional Weyl tensors. Using (4.3) we obtain the Friedmann-like equation

$$
H^{2}=\frac{8 \pi G_{4}}{3} \rho+\frac{C}{a^{4}},
$$

where we have assumed that a metric on the brane is the flat FriedmannRobertson-Walker (FRW) metric with the scale factor $a(t)$. And the energymomentum tensor on the brane is given by $T_{\mu}^{v}=-\rho \delta_{\mu}^{v}$, where $\rho$ is an energy density on the brane. The coupling $\kappa_{5}^{2}$ is related to the Newton constant, $\kappa_{5}^{2} / l \equiv \kappa_{4}^{2}=8 \pi G_{4}$. Equation (4.4) is a Friedmann-like equation for the expansion rate $H(H=\dot{a} / a)$ of a 3-brane embedded in five dimensional bulk. An extra term $C / a^{4}$ ( $C$ is a constant) is known as the dark radiation since it decays in the same way as radiation, the energy density during radiation domination is proportional to $a^{-4}$. Braneworld cosmology is therefore different to the standard cosmology, $H^{2} \sim \rho$. Notice that we recover the standard cosmology for large scale of the scale factor $a$, because we can ignore the dark radiation term. The integration constant $C$ is determined by initial conditions in the 5-dimensional space-time. Its effect corresponds to that of radiation.

Using the junction condition, up to the second order we obtain the effective equation of motion

$$
{ }^{(4)} G_{\mu}^{v}(h)+2 l^{2}\left(\tau_{\mu}^{v}+\alpha S_{\mu}^{\nu}+\frac{\beta}{3} \kappa_{\mu}^{v}\right)=\frac{\kappa_{5}^{2}}{l} T_{\mu}^{v},
$$

where $S_{\mu}^{\nu}$ is given by eq. (3.28) and

$$
\kappa_{\mu}^{\nu}={ }^{(4)} R^{(4)} R_{\mu}^{\nu}-\frac{1}{4} \delta_{\mu}^{\nu(4)} R^{2}-D_{\mu} D^{\nu(4)} R+\delta_{\mu}^{\nu} D_{\sigma} D^{\sigma(4)} R,
$$

and

$$
\begin{aligned}
& \tau_{\mu}^{\nu}=t_{\mu}^{\nu}-\frac{1}{24}\left({ }^{(4)} R_{\mu}^{\nu}{ }^{(4)} R-\frac{1}{4} \delta_{\mu}^{\nu}{ }^{(4)} R^{2}\right)-\frac{1}{32} \delta_{\mu}^{\nu}\left({ }^{(4)} R^{\alpha \beta}{ }^{(4)} R_{\alpha \beta}-\frac{1}{3}{ }^{(4)} R^{2}\right) \\
& -\alpha S_{\mu}^{\nu}-\frac{\beta}{3} \kappa_{\mu}^{\nu} .
\end{aligned}
$$


Here traceless tensor $t_{\mu}^{v}$ is defined by eq. (3.29), $\alpha$ and $\beta$ are constant that represents the freedom of the gravitational wave in the bulk. The trace condition of equation (4.6b) leads to

$$
\tau_{\mu}^{\mu}=-\frac{1}{8}\left({ }^{(4)} R^{\alpha \beta}{ }^{(4)} R_{\alpha \beta}-\frac{1}{3}{ }^{(4)} R^{2}\right)-\beta D^{\sigma} D_{\sigma}{ }^{(4)} R .
$$

We observe that the above equation is nothing but a general form of a four dimensional conformal anomaly which describes the breaking of conformal invariance due to quantum effects (regularization and renormalization), defined on curved backgrounds ${ }^{[14]}$. The coefficient $\beta$ is scheme-dependent constant which can be removed by adding ${ }^{(4)} R^{2}$ term in the effective action. Hence, this part is interpreted as the CFT in the context of the AdS/CFT correspondence. In order to obtain an explicit relation between the geometrical approach (equation 2.30) and AdS/CFT approach, we start by defining

$$
T_{\mu \nu}^{C F T}=-2 \frac{l^{3}}{\kappa_{5}^{2}} \tau_{\mu v} .
$$

Then, we can write the equation (4.5)

$$
{ }^{(4)} G_{\mu \nu}=\frac{\kappa_{5}^{2}}{l}\left(T_{\mu \nu}+T_{\mu \nu}^{C F T}\right)-2 l^{2}\left(\alpha S_{\mu \nu}+\frac{\beta}{3} \kappa_{\mu \nu}\right) .
$$

The next step is to find the evolution equations for Weyl tensor $E_{\mu}^{v}$. This term carries information of the bulk gravitational field on the brane. We get

$$
\begin{aligned}
E_{\mu}^{\nu(2)}= & \frac{l^{2}}{8} \delta_{\mu}^{v}\left({ }^{(4)} R^{\alpha \beta}{ }^{(4)} R_{\alpha \beta}-\frac{1}{2}{ }^{(4)} R^{2}\right)+\frac{l^{2}}{6}\left({ }^{(4)} R_{\mu}^{\nu}{ }^{(4)} R\right)-\frac{l^{2}}{4}\left({ }^{(4)} R_{\mu}^{\rho(4)} R_{\rho}^{v}\right) \\
& +2 l^{2}\left(\alpha S_{\mu}^{\nu}+\tau_{\mu}^{v}+\frac{\beta}{3} \kappa_{\mu}^{v}\right)
\end{aligned}
$$

If we define

$$
P_{\mu}^{V}=\frac{1}{8} \delta_{\mu}^{V}\left({ }^{(4)} R^{\alpha \beta}{ }^{(4)} R_{\alpha \beta}-\frac{1}{2}{ }^{(4)} R^{2}\right)+\frac{1}{6}\left({ }^{(4)} R_{\mu}^{v}{ }^{(4)} R\right)-\frac{1}{4}\left({ }^{(4)} R_{\mu}^{\rho(4)} R_{\rho}^{v}\right) .
$$

We can write equation (4.10) as follows 


$$
E_{\mu}^{v(2)}=l^{2} P_{\mu}^{v}+2 l^{2}\left(\alpha S_{\mu}^{v}+\tau_{\mu}^{v}+\frac{\beta}{3} \kappa_{\mu}^{v}\right)
$$

Inserting equation (4.11) into equation (4.9) yields

$$
{ }^{(4)} G_{\mu}^{v}=\frac{\kappa_{5}^{2}}{l} T_{\mu}^{v}+l^{2} P_{\mu}^{v}-E_{\mu}^{v(2)}
$$

At the lowest order we have the Ricci tensor

$$
{ }^{(4)} R_{\mu}^{v}=\frac{\kappa_{5}^{2}}{l}\left(T_{\mu}^{v}-\frac{1}{2} \delta_{\mu}^{v} T\right)
$$

Substituting this expression into equation (4.10) we obtain

$$
P_{\mu}^{v}=\frac{\kappa_{5}^{4}}{8 l^{2}} \delta_{\mu}^{v}\left(T_{\rho \sigma} T^{\rho \sigma}-\frac{1}{3} T^{2}\right)+\frac{\kappa_{5}^{4}}{12 l^{2}} T T_{\mu}^{v}-\frac{\kappa_{5}^{4}}{4 l^{2}} T_{\mu}^{\rho} T_{\rho}^{v} .
$$

Thus equation (4.12) becomes

$$
{ }^{(4)} G_{\mu}^{v}=\frac{\kappa_{5}^{2}}{l} T_{\mu}^{v}+\kappa_{5}^{4} \Pi_{\mu}^{v}-E_{\mu}^{\nu(2)},
$$

where $\Pi_{\mu}^{v}$ is given by equation (2.33).

We are now able to write the effective action on the brane corresponding to the effective Einstein equation (4.13) is

$$
\begin{aligned}
& S=\frac{l}{2 \kappa_{5}^{2}} \int d^{4} x \sqrt{-h^{(4)}} R+S_{\text {matter }}+S_{C F T} \\
& +\frac{\alpha l^{2}}{2 \kappa_{5}^{2}} \int d^{4} x \sqrt{-h}\left({ }^{(4)} R^{\mu \nu}{ }^{(4)} R_{\mu \nu}-\frac{1}{3}{ }^{(4)} R^{2}\right)+\frac{\beta l^{2}}{6 \kappa_{5}^{2}} \int d^{4} x \sqrt{-h^{(4)}} R^{2},
\end{aligned}
$$

where $S_{C F T}$ is denotes the non local effective action constructed from $\tau_{\mu}^{v}$.

\section{Conclusions}

In this paper, we have investigated the effective equation of motion on the brane following the low energy expansion scheme (gradient expansion method) in the context of RS II systematically. At zero ${ }^{\text {th }}$ order, as we should expect, the equation obtained are just the fine tuning condition of the RS II model between the brane tension and the bulk curvature radius. At first order, the effective 
equation of motion is given by equation (4.3). The resulting effective equations of motion are modified with respect to the conventional Einstein equation due to the presence of a non-local term $E_{\mu \nu}$. Its contribution is fundamental importance as it might be relevant even at low energy. In this case the Friedmann equation on the brane is modified by radiation term.

The effective equations of motion at second order for the Randall-Sundrum II are claimed as an AdS/CFT correspondence described by equation (4.15). We clarify the emergence of the AdS/CFT correspondence by calculating the Weyl tensor at this order.

We also generalize the results for a Randall-Sundrum model, extending the low energy gradient expansion scheme to a multibrane (more than two branes) model and deriving the effective equations of motion in this new scenario ${ }^{[15]}$. The extension to a multibrane scenario is interesting as string theory and Mtheory, which provide the general motivation for studying brane world models, naturally allow for multibrane scenario.

\section{Acknowledgement}

We would like to acknowledge J. Soda (Dept. of Physics, Kyoto University, Japan) for useful discussion about D-branes theories through e-mail correspondences. One of us (Arianto) would like to thank BPPS, Dikti, Depdiknas, Republic of Indonesia for financial support. He also wishes to acknowledge all members of Theoretical High Energy Physics Laboratory, Department of Physics, ITB, for warmest hospitality.

\section{References}

1. Green, M.B., Schwarz, J.H., and Witten, E., Superstring Theory, Vol. I and Vol. II, Cambridge Monographs on Mathematical Physics, Cambridge University Press, (1988);

Zen, F.P., Superstring Theory, D-Brane and Cosmology, Lecture Note (in Indonesia), Theoretical Physics Lab., Department of Physics, ITB, unpublished, (2004)

2. Lukas, A., Ovrut, B., Stelle, K.S., and Waldram, D., Heterotic M-Theory in Five Dimensions, Nucl. Phys. B 552 (1999) 246 [ArXiv: hep-th/9806051].

3. Zen, F.P., Gunara, B.E., and Arianto, Mass Hierarchy Problem in Brane Cosmology, Proc. Seminar MIPA IV, ITB, (2004) 117;

Zen, F.P., Arianto, Gunara, B.E., and Feranie, S., S-Brane Solutions with Accelerating Cosmology in M-Theory, was accepted for publication in Journal of Mathematics and Sciences, MIPA, ITB, (2006). 
4. Randall, L. and Sundrum, R., A Large Mass Hierarchy from a Small Extra Dimension, Phys. Rev. Lett. 83 (1999) 3370 [arXiv: hep-ph/9905221];

Randall, L. and Sundrum, R., An Alternative to Compactification, Phys. Rev. Lett. 83 (1999) 4690 [arXiv: hep-th/9906064].

5. Zen, F.P., Kosasih J.S., and Gunara, B.E., Hierarchy Problem and RandallSundrum Mechanism in D-Brane Theory, Proc. Annual Physics Seminar, Oct. 2, 2003, ITB, Bandung (2003).

6. Khoury, J., Ovrut, B.A., Steinhardt, P.J., Turok, N., The ekpyrotic Universe: colliding branes and the origin of the hot Big Bang, Phys. Rev. D 64 (2001) 123522 [arXiv: hep-th/0103239];

Tolley, A.J., Turok, N., and Steinhardt, P. J., Cosmological perturbations in a big crunch/big bang space-time, Phys. Rev. D 69 (2004) 106005, arXiv:hepth/0306109].

7. Gubser, S.S., AdS/CFT and Gravity, Phys. Rev. D 63 (2001) 084017 [arXiv: hep-th/9912001].

Anchordoqui, L., Nunez, C. and Olsen, K., Quantum Cosmology and AdS/CFT, Journal of High Energy Physics, 10 (2000) 050 [arXiv: hepth/0007064].

8. Zen F. P., Gunara, B.E., Arianto, and Zainuddin, H., On Orbifold Compactification $N=2$ Supergravity in Five Dimensions, Journal of High Energy Physics 08 (2005) 018 [arXiv: hep-th/0407112].

9. Shiromizu, T., Maeda, K., and Sasaki, M., The Einstein equation on the 3Brane World, Phys. Rev D 62 (2000) 024012 [arXiv: gr-qc/9910076].

10. Langlois, D., Brane cosmology: an introduction, Prog. Theor. Phys. Suppl.148 (2003) 181 [arXiv: hep-th/0209261];

Brax, P. and van de Bruck, C., Cosmology and brane worlds: A review, Class. Quant. Grav. 20 (2003) R201 [arXiv: hep-th/0303095].

11. Marteen, R., Brane-World Gravity, Living Rev. Rel. 7 (2004) 1 [arXiv: hepgrqc/0312059] and references therein.

12. Kanno, S. and Soda, J., Brane World Effective Action at Low Energies and AdS/CFT Correspondence, Phys. Rev. D 66 (2002) 043526 [arXiv: hepth/0205188];

Kanno, S. and Soda, J., Radion and holographic brane gravity, Phys. Rev. D 66 (2002) 083506 [arXiv: hep-th/0207029].

13. Wald, R. M., General Relativity, The University of Chicago Press, (1984) p. 40.

14. Birrel, N.D., Davis, P.C.W., Quantum Fields in Curve Spacetime, Cambridge Monograph on Mathematical Physics, CUP, 1982;

Duff, M.J., Twenty Years of the Weyl Anomaly, Class. Quant. Grav. 11 (1994) 1387 [arXiv : hep-th/9308075].

15. Zen F. P., Arianto, Gunara, B.E., and Zainuddin, H., The Low Energy Effective Equations of Motion for Multibrane Worlds Gravity, to be submitted for publication [arXiv : hep-th/0511257]. 